I must confess to a feeling of astonishment that anyone in the position of the Editor should gravely hint that, if called upon to advise how to combat tuberculosis, we might practically ignore the influence of breeding, or of the manner of housing young stock. He would say, "go on with your old methods as long as you like, only avoid contagion."

How does he reconcile this again with the finding of the London Congress, with regard to human tuberculosis, but which is quite as applicable to stock?

"That in the opinion of this Congress overcrowding, defective ventilation, damp and general insanitary conditions, diminish the chance of curing consumption, and aid in predisposing to, and spreading the disease."

To sum up,-In dealing with tuberculosis it should be our endeavour to get rid of all clinically diseased and reacting stock, and in this both I and the Editor are agreed; and then, but here we appear to differ, to encourage the breeding, and bringing up under sound hygienic principles, of healthy stock, not paying so much attention to artificiality, as to more natural environments, and I believe that we could in this manner check the bacilli. I should not have the remotest fear for myself, for example, that, given my present healthy, out-of-door life, tubercle bacilli could make any impression on my body, though they might be about me.

It does not follow that, because in the dressing of surgical wounds we can prevent the ingress of pathogenic organisms, we are to conclude that they are all destroyed; no, they are very much in evidence, as we know to our cost if we are the least negligent in our treatment.

So with these bacilli, tubercle, and others; they are not all going to be destroyed just yet, but we should endeavour to make it as difficult as possible for them to attack successfully our cattle, and one way of doing this is by keeping the latter healthy and vigorous.

\title{
MALIGNANT JAUNDICE IN THE DOG.
}

\section{By William Robertson, M.R.C.V.S., Government Veterinary Surgeon, Cape Colony.}

ThIs disease, variously named malarial fever, malignant malarial jaundice, and by some dog-owners simply distemper, is one of the most fatal diseases of the dog met with in Cape Colony.

It may be roughly defined as a malarial disease affecting dogs, communicable by direct inoculation from animal to animal, but not by cohabitation or ingestion, and due to the presence of a hamatozoon in the red blood corpuscles.

The disease attacks Cape bred and imported dogs indiscriminately, is confined to certain parts of the country, and is most prevalent in the summer and autumn.

Judging by contemporary literature on the subject, there can be no doubt that this disease is met with affecting dogs in other countries. Celli, in his work on "Malaria," alludes to Pointer dogs brought from Lombardy to the Compagna marshes contracting a form of malaria, and mentions the presence of an intracorpuscular body in the red 
blood corpuscles. In I 890 Piana and Galli Valerio pointed out in the blood of the dog in Italy an intraglobular hæmatozoon resembling in form the pyrosoma bigeminum of Smith and Kilborn. At the Societé de Biologie, 2oth January I900, M. P. Leblanc of Lyons Veterinary College evidently describes a case of this disease under the heading "Nature of Infective Icterus in the Dog," as he alludes to a hæmatozoon analogous to that found in red water in the ox. Professor Koch has seen the double pyrosoma in the blood of dogs in East Africa, and Dr Marshon verified its existence in Senegal in the blood of eleven dogs. Lastly, in the Recueil de Médécine Vettininaire (reprinted in the Veterinary Journal, I90I) Messrs Nocard and Almy in France describe a case which in clinical history, symptoms, and microscopical appearance of the blood, resembles a description of a good case of malignant jaundice of the dog in Cape Colony.

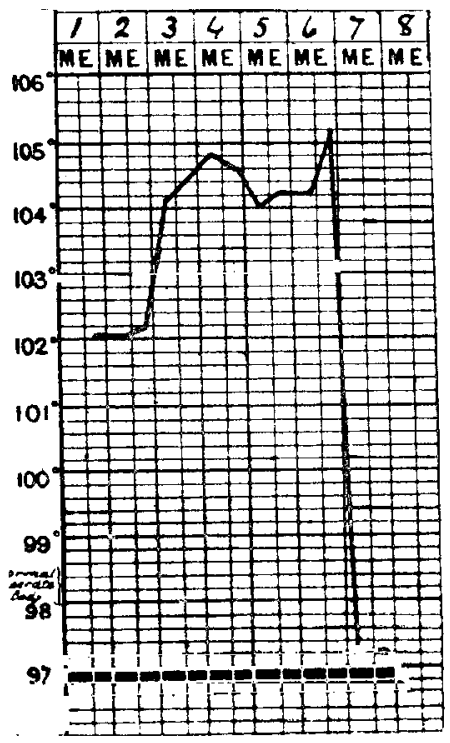

FIG. 1.

Temperature chart of a case of malignant jaundice in a dog inoculated intravenously with 5 ce. of blood' (from a dog naturally infected).

Symptoms.-In a case produced by subcutaneous inoculation of a healthy animal with infective material, the temperature rises on the third day, and death supervenes on the twelfth or thirteenth. If, on the other hand, the inoculation is intravenous, the initial rise of temperature still occurs on the third day, but death takes place much sooner-from the eighth to the ninth day, or even earlier.

The first clinical symptoms are noticed on the third day after inoculation ; the dog is dull, listless, drowsy, refuses food, and may be thirsty. On the fourth day in a case of intravenous inoculation you see the biliary staining of the visible mucous membranes commencing, just a faint dirty-yellow being noticeable. During the following $d$ ay the characteristic hæmoglobinuria may make its appearance, the uri ne 
being in many cases as dark as claret. All the visible mucous membranes are of an almost chrome-yellow colour, and any naked skin, such as flanks, prepuce, inner edge of ears, etc., shares in the staining. The animal lies perfectly helpless; the whole body exhales a foul odour, constipation is commonly present, the tongue is foul and furred, the teeth dirty, and there may be shedding of part of the gums down to the alveoli. The animal in most cases appears to die from collapse and weakness, due to the drain upon the blood produced by the hamoglobinuria. That this is not alzeays the cause of death I am sure. Just as in Texas fever (or red water as it is called in Cape Colony) one may get an animal dying with all the symptoms of that disease except the hæmoglobinuria, and find the typical pyrosoma in the blood, so one may get a dog with all the other symptoms of malignant jaundice except hromoglobinuria, and, as in the case of the ox, recover the special pyrosoma from the blood.

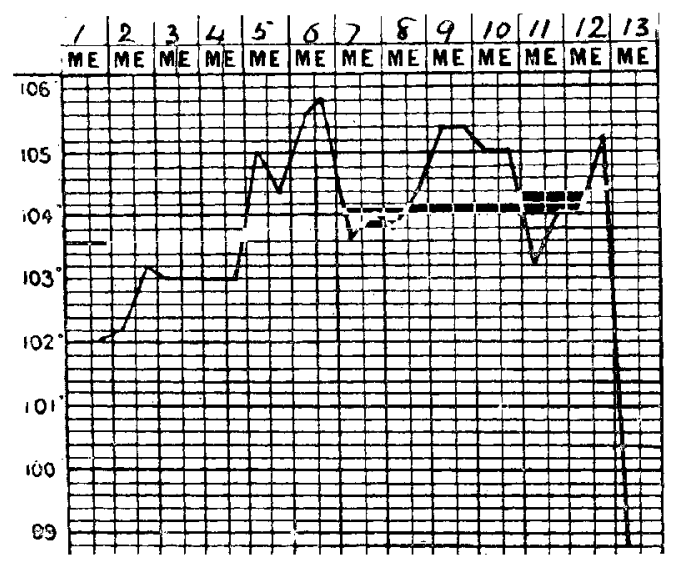

FIG. 2.

Temperature chart of a case of malignant jaundice in a dog inoculated subcutaneously with 5 cc. of blood (from a dog naturally infected).

If the case of jaundice has been produced by subcutaneous injection the course of the disease is slower, but the termination and the general train of symptoms are the same.

Post-mortem Appearances.-I take two cases at random from the post-mortem book.

CASE I. 2oth October. - Subject-A small smooth-haired foxterrier dog; died during night; inoculated on the 3 th inst. with $5 \mathrm{cc}$. blood intravenously from a case of naturally contracted disease in a collie. Body emaciated, weight Io lbs. 4 ozs.; eyes sunk in the head; visible mucous membranes and bare places on the body, such as the prepuce, flanks, etc., of a dirty-yellow colour ; tongue heavily furred ; teeth covered with dirty yellowish-brown deposit, possessing a most fotid odour; subcutaneous fat, subcutaneous connective-tissue, and superficial muscular tissue stained yellow; intraperitoneal fat ditto; heart shows petechiæe on epicardium and endocardium; liver much 
enlarged, friable, yellowish-red in colour, weight 3 Io grammes; spleen very much enlarged and distended, pulp much softer and darker than normal, weight 6 I grammes ; stomach quite empty, congested at fundus, mucous membrane generally bile-stained; small intestines bile-stained; large intestines inflamed throughout, with copious exudate of a rustyred colour on the mucous membrane, no ulcers visible ; kidneys externally bile-stained, and on section paler than normal in the cortex; bladder contained about $50 \mathrm{cc}$. of dark claret-coloured urine, with a foetid odour. Microscopically the blood and spleen showed the presence of the characteristic pyrosoma.

CASE II. I7th October.-Subject-A small, smooth-haired, foxterrior dog. This was a case of natural infection, first noticed sick on $7_{\text {th }}$ October, was not treated during the course of the disease, and died on the morning of $17^{\text {th }}$ October. Body in good condition, weight $10 \mathrm{lb}$; all visible mucous membranes and skin of belly stained yellow; subcutaneous tissues and fat of a bright yellow colour; heart normal; liver much congested and enlarged, more friable and

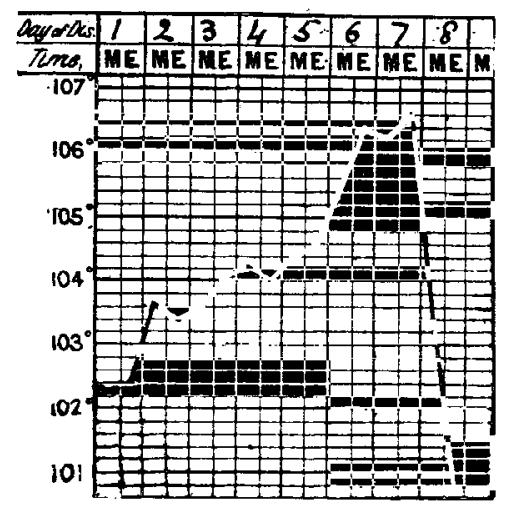

FIG. 3.

Temperature chart of a case of malignant jaundice in a dog inoculated intravenously with 5 cc. of blood (from a dog infected by inoculation).

darker in colour than normal; spleen much enlarged and softened; stomach had mucous membrane congested, and contained about an ounce of a dark coffee-coloured liquid; small intestines normal; the large contained slightly brown or rust-coloured mucus; no ulcers anywhere; kidneys normal: bladder full of claret-coloured urine. Microscopically the blood showed the presence of a double pyrosoma in the red corpuscles.

From a study of the post-mortem records of these two cases, one naturally infected, the other produced by inoculation with infective material, it will be seen that the post-mortem appearances in the two cases were very similar. The most striking features in the post-mortems I have made are, the deep bile-staining of skin and mucous membrane, the enormous increase in size of the spleen, the blood-coloured urine, and the peculiar odour envolved from a living or freshly killed dog badly affected with the disease. The increase in size of the spleen is perhaps the most marked post-mortem lesion. 
Causal Parasite.- This is without doubt a double pyrosoma, with, in the complete stage, the thin ends approximated, and resembling in its general appearances the pyrosoma bigeminum of Texas fever. This pyrosoma in the dog was first demonstrated in South Africa by Dr Carrington Purvis, at the Bacteriological Institute, Grahamstown. A few months afterwards Mr Spreull, M.R.C.V.S., one of the Government veterinary surgeons, proved the inoculability of the disease. $\mathrm{He}$ says, "Intravenous inoculation I found produced acute symptoms in about four days, and death about the sixth; subcutaneous inoculation, on the other hand, was much slower, death occurring from the eleventh to the thirteenth day, after two or three days' illness." Since then I have made extensive inoculation experiments and post-mortem examinations in connection with this disease. On one occasion I obtained blood from a sick dog, and kept up the disease through thirteen animals, every one of which succumbed.

I find the intracorpuscular bodies mentioned by Dr Purvis to be

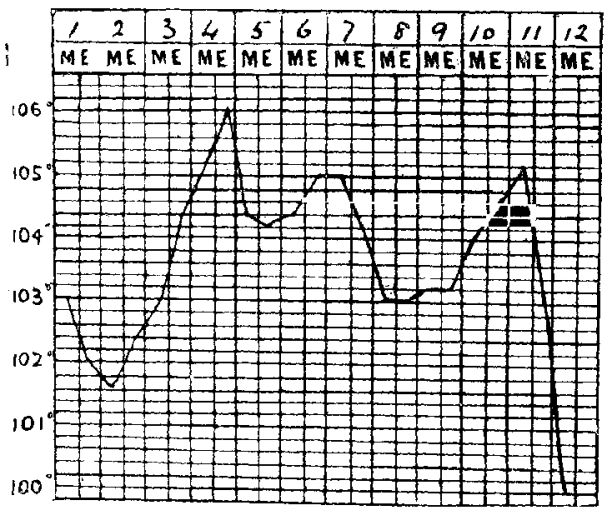

Fig. 4.

Temperature chart of a case of malignant jaundice in a dog inoculated subcutaneously with 5 cc. of blood (from a dog infected by inoculation).

present in every case of the disease met with. The blood is infective on the third day, and they make their appearance in the red corpuscles on the fourth day after subcutaneous inoculation with virulent blood. I have also found the pyrosomata free from the corpuscles in a very advanced stage of the disease.

The pyrosomata are met with in smears from liver and spleen, and the latter organ in a fatal case positively swarms with them, one corpuscle having as many as five or six bodies in its interior.

Though in many ways strongly resembling the pyrosoma bigeminum of Texas fever, the intracorpuscular bodies in malarial jaundice in the dog present certain well-marked differences. They are larger, and not so markedly pyriform as the Texas fever parasite, and present somewhat of an oat shape, staining slightly in the centre, which possesses one clear spot, and more deeply at two or three places in the margin. This parasite stains well by carbol thionin blue, methylene blue, and the double stains of Plehn and Czinz-is-kis, but 
does not take up the hæmatoxylin when using Cole's method of eosin and hæmatoxylin. It is found in sections of tissues, and blocking the capillaries in the brain. In some cases single bodies in a corpuscle may be met with, and I do not think they appeared only when the blood was examined in the early stage of the disease, as I have single pyrosomata in specimens taken at post-mortems. Using the blood of a case which showed the pyrosoma microscopically, I was unable to infect the horse, sheep, ox, cat, fowl, guinea-pig, rabbit, rat, or mouse.

Therapeutic Remedies. - While keeping up the strain of blood for inoculation experiments, I had an opportunity of trying therapeutic remedies, with marked ill success, but I do think the disease was aggravated, and rendered more virulent, by passage through dogs. All sorts of remedies likely to have a beneficial effect were triedquinine, calomel, ammon chlor, ext. belladonæ, carbolic acid, benzoate of soda, alone and in combinations, without very satisfactory results.

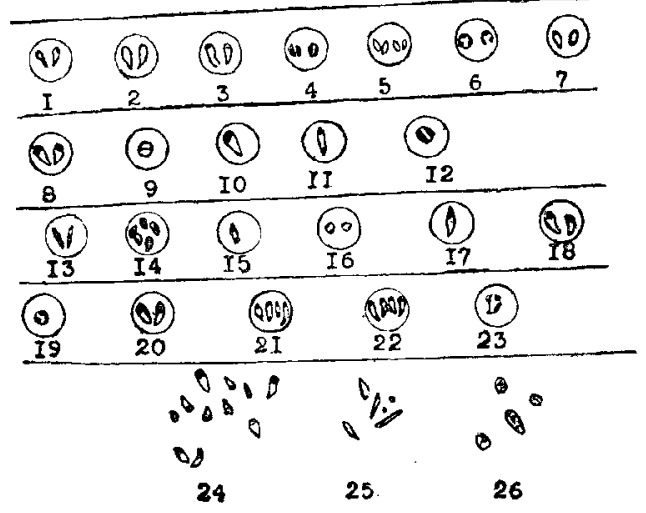

FIG. 5.

Various forms assumed by the causal parasite of malignant jaundice in the dog.

Nos. 2, 8, and 20 are the forms most commonly met with. Nos. 24, 25, and 26 are parasites free in the blood-stream.

I certainly think carbolic acid hastened death. The treatment giving best results was a calomel pill to start with, then a calomel and quinine pill four times a day (the calomel must be given in very large doses). Good nursing and careful diet are, of course, essential to a satisfactory termination of the illness.

Means of Infection.-Having settled the causal parasite of this disease, one naturally next desires to find out the bearer of this cause of infection. At present we have only the history of cases of natural infection and the analogy of the spread of another disease associated with a pyrosoma to guide us to any decision in the matter; and, judging from the history of many cases of malignant jaundice, 1 feel little doubt that the infection in this disease can also be conveyed by ticks. I will give a couple of cases of natural infection, as sort of circumstantial evidence. 
CASE I. Subject-A small rough-haired mongrel dog, kept for the past two months in close confinement in a glass case, and used by $\mathrm{Mr}$ Chas. Lounsbury, the Government Entomologist, as a host for rearing certain ticks from various domesticated animals.

This dog became ill, and presented all the symptoms of malignant jaundice, with pigmentation of visible mucous membranes, and presence of pyrosomata in the blood. The dog eventually died, and the post-mortem appearances, macroscopical and microscopical, were typical of a case of that disease.

Mr Lounsbury tells me that some time before the dog fell sick he obtained from some dogs in the neighbourhood of Cape Town (which dogs were said to be salted or immune to malarial jaundice) a few dog ticks, and at the same place and time secured one tick from an animal affected with the disease. These ticks were hatched out in the Laboratory; during the process the tick from the sick dog got missed and the larval ticks placed for the purpose of feeding upon the rough-haired $\mathrm{dog}$ in the case. I feel certain that larva of the tick from the dog suffering from malignant jaundice got mixed with the larvæ of the salted ticks and so produced the disease, as we know larvæ from ticks off salted animals will not do so.

CASE II. Subject-A small aged Pointer dog, the property of a gentleman in the suburbs, who had kept this dog at his house for some two years.

History.-This dog, which, by the way, the owner said had had this sickness before, was taken on a shooting trip to Kuils, a very bad tick veldt, ten days before, returned with ticks on him, was first noticed sick three days ago, and had rapidly got worse.

When I saw the animal it was lying prostrate, unable to stand or move. Mucous membranes of eyes and mouth, the naked skin of the flanks, even the internal surface of the ears were stained a deep dirty yellow, and there was a drop of dark blood-coloured urine on the tip of the prepuce. The teeth were covered with sordes, and the tongue with a thick yellowish-green fur; the mouth had a most disagreeable odour.

I took blood from the jugular vein, and this under the microscope proved a most curious specimen, as coverglass after coverglass was examined without finding a complete red corpuscle, but every field teemed with pyrosomata free, some lying with their thin ends approximated, as seen in the red corpuscles, while others appeared singly or in groups of three or four. I stained with Plehn's and eosin, then methylene blue, and in all the covers not a single red corpuscle took the eosin stain, all that remained of them being some granular débris.

The dog lived twenty-seven hours after the abstraction of this sample of blood.

The inference I drew from the above case was as follows:-

The neighbourhood of the dog's home is not a tick veldt, and the animal is taken to a tick veldt, and to a neighbourhood where malignant jaundice is known to exist, returns with tick attached to his skin, and shortly after succumbs to this disease. These facts being so, I do not think that it is too much to say that the dog contracted the disease from the ticks at Kuils River.

In conclusion, I should like to point out that I have seen cases of 
jaundice affecting dogs in England in which the mucous membranes and even the skin showed bile-staining, and I think it would be worth while for some of the members of our profession to make and examine a few coverslips of blood from the next case they meet.

I have attached to these notes four temperature charts, showing the characteristic temperature curve in this disease, whether produced by subcutaneons or intravenous infection. It will be noticed that the curve in a dog infected from a case of natural infecti : he same as when the infecting material is taken from an artificially produced case.

\section{THE PART PLAYED BY THE DOG TICK, “HAMAPHYSALIS LEACHI," AS A CARRIER OF INFECTION IN MALIGNANT JAUNDICE OF THE DOG.}

The fact that certain external parasites can and do carry infection from a sick to a healthy animal is, I think, acknowledged by most authorities. Smith and Kilborn have shown that the progeny of ticks, rhipicephalus annulatus (Say), act as carriers in the case of Texas fever or red water in cattle. The value of such knowledge is apparent, as by its use we are able to minimise the spread of such diseases, e.g., we spray cattle to kill ticks before changing pasture, and prevent them carrying Texas fever to a clean area, and cover the pools with kerosene to destroy the young of the malaria-bearing mosquito.

The following experiments, conducted by $\mathrm{Mr}$ Charles Lounsbury, Government Entomologist, and myself, throw some light upon the dog tick as a carrier of infection in the disease known in this country as malignant jaundice of the dog. I have previously mentioned two cases of malignant jaundice, and the chain of evidence which prompted me to state that I considered the dog tick to be capable of conveying the contagion of that disease. It will now be seen from the experiments detailed below that artificially hatched ticks, the progeny of females matured on affected animals, can produce the disease in healthy dogs; but only when they reach the adult stage.

From specimens submitted to him, Professor G. Neumann, of the Veterinary School, Toulouse, identified the South African dog tick as hamaphysalis Leachi; it was this tick which we used.

The brood ticks from which the eggs, and later the young ticks employed in the following experiments, were obtained, came from a naturally infected $\operatorname{dog}$ - a mongrel, alluded to in the previous notes, and quoted as a case in support of the tick-borne theory of the disease. These ticks laid eggs, which were hatched out on moist sand in an incubator. The dogs used for the first experiment were two fox-terrior puppies, which from birth, and till the termination of the experiment, were kcpt in such isolation as to preclucie the chance of natural infection. When ten days old, the dogs were placed under observation; temperatures were normal, and no parasites could be found in the blood. When about a month old, on I 5 th and zoth June, both puppies were infested with larval (newly hatched) ticks. The temperatures were now regularly taken, and the blood examined at frequent intervals, but no fever manifested 'itself, and no parasites could be found in the blood. 
In July the same dogs were again infested with a batch of the same ticks, which had moulted and entered the nymph stage; again no result followed. On 27 th August the same dogs were infested with a batch of the same ticks as before, but now in the adult stage. The dogs began to look sick on $9^{\text {th }}$ September, temperature rose to $105^{\circ}$, and on examination of blood films the red corpuscles teemed with parasites, some corpuscles containing as many as eight of the pearshaped forms. These intracorpuscular bodies varied in shape, some being spherical or ring shaped, with a darkly-staining wall, and some thickening of this, or condensation of protoplasm, at opposite sides of the circle; others were typical pyrosomata, two being the most frequent number noticed, but one, and up to eight, were also found in a single red corpuscle. Some parasites were found free outside the blood corpuscles, both spherical and pyriform in shape, and even clove-like, that is, the thickened portion becoming suddenly thinner, instead of tapering like an oat seed. Some of the intracorpuscular spherical forms, which had a deeply-stained portion at either side of their periphery, seemed to have a fine partition dividing them in two. The puppies died on the I 4 th and I 5 th days, respectively, after infection with the young fully-formed ticks.

Post-mortem appearances were those of typical malignant jaundice. Liver much enlarged and congested; spleen large, very soft, and dark in colour; blood thin, coagulating imperfectly; a few soft clots in the right ventricle, veins empty; subcutaneous tissues and peritoneal fat bile-stained ; urine colour of claret and water ; kidneys enlarged, pink in colour, rather firm, and on section glistening like paraffin wax.

I am certain :-

(I). That the dog from which the brood female ticks were taken in the first instance was suffering from the disease, as the microscopical examination of the blood during life showed numerous intracorpuscular bodies, and a post-mortem examination confirmed the diagnosis.

(2). That the hatched-out progeny from these brood ticks produced malignant jaundice in the two puppies, from which disease they died.

Why the puppies did not contract the disease when infested with the ticks in larval or nymph stages admits of two explanations:either (a) very young dogs may be naturally immune, up to a certain stage, or $(b)$ very young ticks from infected brood females are unable to produce the disease. To ascertain which was the correct explanation, I performed the two following experiments :-

(I). One of the sick puppies was bled at the height of the disease (temp. $105^{\circ} \mathrm{F}$. and many parasites in the blood), and $3 \mathrm{cc}$. was injected into the jugular vein of a healthy twelve-months old dog on 9 th September. Intracorpuscular bodics appeared in the blood. on the I th, on the 15 th the temperature rose to $105^{\circ} \mathrm{F}$, and the animal died from malignant jaundice on the I 8 th.

(2). A puppy fourteen days old (one of eight), sucking its mother, was also inoculated with a minute dose of the same blood on 9 th September. Intracorpuscular bodies appeared in its blood on I 4 th September, temperature $103^{\circ} 2^{\circ}$, and death from malignant jaundice took place on 2oth September.

The blood of both animals was examined previous to inoculation, and found to be free from pyrosomata.

As confirmatory evidence that dog ticks, the progeny of infected 
females, can only convey the disease during the adult stage of their growth, a second series of experiments was undertaken. Dogs Nos. $5,6,7$, and 8 were selected, and kept under such circumstances that natural infection was an impossibility; for some time previous to infesting them with ticks their temperatures were carefully registered and their blood frequently examined.

Dogs Nos. 5 and 7 were infested with larval ticks, the progeny of infected females. The temperatures were taken regularly, and no rise was noticed; films of their blood were taken at frequent intervals, and no parasites could be seen. These two dogs are alive and well at time of writing.

Dogs Nos. 6 and 8 (a puppy and a full-grown dog respectively) were infested with ticks in the adult stage, the progeny of infected females, on 23 rd September. On the 4 th October the temperature of No. 8 was noticed to be above normal. Films from the blood were at once examined, and the causal parasite of malignant jaundice found to be present in the red corpuscles. On 5 th October the temperature of $\operatorname{dog}$ No. 6 showed a rise, and microscopical examination of the blood revealed the presence of the typical parasite.

That artificially-hatched adult ticks, the progeny of infected females, can produce this discase in healthy animals, I think must be admitted.

Age does not appear to give any protection from this disease, as in previous experiments I have been able to produce it by subcutaneous and intravenous inoculation in dogs of all ages, and with the utmost certainty.

In conclusion, it may be added that large quantities of dog's blood, containing the parasites in large numbers, have been injected intraperitoneally into guinea-pigs, pigeons, etc., without producing death, or even any lasting constitutional disturbance. This experiment was undertaken to ascertain if the parasite of malignant jaundice in the dog produced the caustic and irritant effects said to follow an intraperitoneal injection of Texas-fever blood, as stated by M. Lignière in his report on that disease in Argentina.

\section{THE VETERINARY INSPECTION OF IMPORTED AMERICAN HORSES,}

By Percy T. Howard, F.R.C.V.S., London.

IN this age of veterinary medical associations, periodicals, new works, etc., it is a difficult task to find a subject of a comparatively unhackneyed character. The busy practitioner has, owing to the need for preparation, little time or opportunity for the performance of experimental or research work, on which to base a scientific communication. Original views on well-thrashed-out points demand for their production an ability almost akin to genius. However, the material utilised in this paper is original, at least with regard to its source.

The inspection has been conducted in the combined interests of the shipowners and the underwriters of the horse insurancc, with a view to taking any steps, or submitting of any recommendations, that 